\title{
HIV/AIDS in Yoruba Perspectives: A Conceptual Discourse
}

\author{
Aderemi Suleiman Ajala \\ Department of Archaeology \& Anthropology, University of Ibadan, Ibadan, Nigeria \\ E-mail: asajala@yahoo.co.uk
}

\begin{abstract}
KEYWORDS HIV/AIDS; sexuality; poverty; Yoruba culture
\end{abstract}
\begin{abstract}
Since 1980s when HIV/AIDS became a worldwide epidemic, the explanation of its aetiology and etymology has established three main global perspectives. These are cultural, dependency, and rational choice theses. These theses have been used to explain the prevalence, and impacts of HIV/AIDS especially in Africa. They have also been used to develop policies against HIV/AIDS. There is a continuous increase in cases of HIV/AIDS in Nigeria especially in the Yoruba dominated States of Southwestern Nigerian. In the region, it is on record that the prevalence was $4.6 \%$ on the average as at 2004; yet those three main global perspectives still govern the explanation and policy intervention of HIV/AIDS. This study therefore examines the application of these theories in Yoruba culture vis-àvis the people's perception of HIV/AIDS. It also explores the possibility of using the theories to establish intervention policies against HIV/AIDS in the study area. The study involves ethnographic fieldwork conducted in some selected communities of the Yoruba society. In-depth interview, key-informant interview, focus group discussions and participant observation were used as data collection strategies. The study revealed that with regard to the Yoruba culture of sexuality, disease, and poverty, the three main global theses have ignored specific internal factors in explaining AIDS pattern in the Yoruba society. Thus, policies emanating from these theses have failed to adequately curtail the prevalence of HIV/AIDS in the society. Hence, there is need for re-direction to community - specific explanation of HIV/AIDS.
\end{abstract}

\section{STUDYBACKGROUND}

For about three decades of the outbreak of HIV/AIDS epidemic, It has caused a lot of devastations to both material and human values in all human societies. Impacts of the epidemic on Gross Domestic Production (GDP) are noticed on the economic decline of many countries especially in Sub-Saharan Africa (Bloom,et al 2004 a). The economic impacts become noticeable as a result of declining and weak labour due to morbidity resulting from the epidemic. Similarly, both the household and national incomes are being diverted to the production of drugs and health-related services almost to the exclusion of other diseases, such as malaria and complications of childbirth. Thus, the outbreak of HIV/AIDS has caused increase in global and national budgetary allocation to the health sector at the expense of other social needs. Similarly, mortality rate has also tripled due to the scourge of HIV/ AIDS. Life expectancy has also declined, and the challenges of social support and care for the less privileged such as orphans, widows, and the aged has doubled due to the incidence of HIV/AIDS.

In the view of above global problems induced by HIV/AIDS, since 1980s global society has been concerned with the actions against the epidemic.
At first, that is, between 1980 and 1990 it was the age of ignorance about the epidemic. Then, there were little understandings about the conception, perception and the implications of that disease across the globe. Every society was trying to reject the ownership of the epidemic, and trying to establish justification theory of rejection of the epidemic. Even the societies with the evidence of homosexuality do not accept the ownership of HIV/AIDS, while those societies with widespread immoral sexuality and breakdown of sexual norms, equally detest the ownership of the disease.

Between 1990 and 2000, there came the age of reasoning. This period featured coming together of the North and the South; developed and developing; and dictatorial and democratic countries to establish basic understanding about the disease in relation to the knowledge, attitudes, and behaviours causing the disease, leading to its spread, and its overall implications on humanity. Of course, this period also witnessed reasoning on how to manage the disease. Thereafter, the post millennium era witnessed another epoch in HIV/AIDS discourse. It is the age of discoveries, which feature(d) biological, cultural, social, and technological discoveries against the causes and spread of HIV/AIDS; and against the devastation of human body system by the disease. 
In some societies especially, the developed societies, there is a dawn of intervention on HIV/ AIDS, albeit, the cure is yet to be established. In African society, the intervention is yet to be witnessed.

Explaining the above developmental phases of HIV/AIDS, and establishing better understanding of the causes, spread, prevalence and effect of the disease on the society, are the three main global theses:

(i) Cultural explanation; (ii) Dependency; and (iii) Rational Choice Explanations (RCE).

The major proponents of cultural explanation include Cadwell, Cadwell et al. (1989) and Odebiyi and Vivekananda (1991). The major construct of cultural explanation is sexual behaviour being the ipso facto cause of HIV/AIDS. The thesis further made a distinction in the pattern of transmission between Africa and the countries of the West as been different because of the "level of sexual activity and the sexual orientation" (Cadwell et al., 1989). In their earliest study on the prevalence of HIV/AIDS, Cadwell et al. (1989) examined the sex ratio, that is male and female ratio on the prevalence of HIV/AIDS. They attributed the rapid spread of the epidemic to the degree of sexual permissiveness in societies. To them, the degree of sexual permissiveness is higher in women especially in Africa where, there is absence of moral and instructional constraints on sexuality. They are of the opinion that Africans hold more permissive attitudes towards sexual relationships with multiple partners and extramarital relationship. They also argued that a pragmatic attitude exists in Africa toward female sexuality, with a fair degree of permissiveness toward premarital relation, which are not too blatantly public, and a degree of acceptance that surreptitious extra-marital relations are not the high point of sin and usually should not be severely punished (Cadwell et al., 1989: 189). In addition, they submitted that African society recognizes the phenomena of longer-term girlfriendship, mistresses and outside wives, who serve in the urban areas as alternatives to polygynous married wives. In their opinions, Odebiyi and Vivekananda (1991) expatiated on the cultural explanation theory by attributing the female sexual permissiveness to the institutions of polygyny, which drives women to seek for sexual fulfilment outside marriage; polyandry, and the high premium placed on having children in African culture as drives for indiscriminate sexual activities. Among the Masai people in East Africa female sexual permissiveness is linked with HIV/ AIDS. The people allow women to be jointly shared by men, who are circumcised the same day and this is said to account for the prevalence of HIV/AIDS. All that is required is that, the "visiting comrade" puts his spear outside the hut to announce his presence, thus he is entitled to the same conjugal right as the husband. This practice is taken to explain the cultural Explanation theory.

Dependency emphasises that AIDS be understood in its wider context of national, regional, and global economic inequalities. According to Schoeff (1992), the spread of HIV/ AIDS is determined by international political economy, social structures and the actions of individuals and groups, which are historically constructed into a system. In his opinion, relying on Schoeff ideas (1992), Hunt (1989) observes that cities are hives of manufacturing, job opportunities, and commercial activities, to the neglect of the rural communities. Thus cities attract people from rural communities, and causing rural poverty. Female migrants in towns who have no jobs turn to prostitutes thereby promoting the spread of HIV/AIDS. Expatiating on this thesis, Ajala (2004) analyses the implication of rural poverty on women's vulnerability to HIV/AIDS. According to him, poverty limits women's choice of fertility and sexual activity; and access to community resources such as education, capital, and income earning probabilities. Thus, making women to depend on their men for a living. Men exploit the situation by negotiating for sex from women. Since many women remain jobless, uneducated and poor, many of them fall prey to indiscriminate sex activities, and are exposed to HIV/AIDS.

Rational Choice Explanation (RCE) is a simplified set of assumptions arising from human behaviour, whereby social action is regarded as a sum total of individuals in terms of costs and benefits (Phillipson and Posner, 1995). To them, sex and determinant of sexuality is a calculation of costs and benefits by individuals in a bid to maximise their interests. The spread of HIV/AIDS through indiscriminate sex has to do with rational choices made by individuals as to the costs and benefits of such risky sex. Such rational choices are influenced by individuals' personal decisions. The RCE specifically focuses on three factors, which can best explain the prevalence of HIV/ AIDS in Africa. These are the nature and size of 
high prevalence of prostitution; and nonmonogamous sexual activity; the high prevalence of sexually transmitted diseases' and the real costs of condom in the African context.

Emanating from the above three global theses are certain actions against the prevalence of HIV/ AIDS. Specifically those actions are the Abstinence from sex, Be a faithful partner, and use of Condom (ABC of HIV/AIDS). These have being the global actions against the spread of HIV/AIDS, which have continued to be promoted. Yet, in many African societies especially in the Yoruba community of Southwestern Nigeria, HIV/ AIDS is still reported to be a major killer disease infecting on the average about 800 people out of the society's population of 46 million daily. The average prevalence is $4.6 \%$ of the total population in that society which is made of six states in Nigeria (NPC, 2001; FMOH, 2002; Osotimehin, 2003).

Thus, pertinent questions arising from the above are: (i) What are the specific cultural perspectives of HIV/AIDS prevalence in the Yoruba society? (ii) How best can the global perspectives explain the prevalence of HIV/AIDS in that society? (ii) To what extent have the initiated strategies worked to prevent the prevalence of HIV/AIDS in the Yoruba society? (iv) How can the incidence of HIV/AIDS in the Yoruba society be theoretically explained? and (v) Can such theoretical explanation be translated to actions against HIV/AIDS in that society?

To answer the above questions, this study involved a qualitative ethnography conducted in four selected communities in the Yoruba society of Southwestern Nigeria with a view to examine the Yoruba perspectives of HIV/AIDS in conceptual.

\section{STUDY METHODOLOGY}

This present study is qualitative, conducted in Oyo and Osun States in the Yoruba society of Southwestern Nigeria, where four study communities were purposively selected. These communities were Ibadan and Kishi in Oyo State and Ilesa and Ile-Ogbo in Osun State. Kishi and Ile-Ogbo are rural communities, while Ibadan and Ilesa are cities. This study began in 2000 A.D. and lasted till 2003. It is a multidisciplinary research and the broad theme is "Cultural Context of HIV/AIDS in the Yoruba Society". The study involved an Anthropologist and nurse as the principal investigators and a sociologist as a research assistant.

Field investigation involved the use of keyinformant interview, In-depth interview with the use of household structured interview, Focus Group Discussion (FGD), Case Study Analyses, and Non-participatory observation. The study population comprised of four hundred and twenty-three PLWAs found in two nongovernmental organisations (NGOs) and hospitals in Ilesa and Ibadan. Some of them were engaged in case analysis. Another set of 200 respondents was selected for the study. These respondents included the public health officers dealing with HIV/AIDS in the hospitals, ministries and NGOs; and People Affected By AIDS (PABAs).

The study perspective involved the combination of both emic and etic perception. This perspective guarantees a comparison of both internal and external factors influencing the understanding of HIV/AIDS and capable of spreading HIV/AIDS infection in the society. The study adopted both individual and community as its unit of analysis. The analytical component of data analysis is a summary of the data, which involves the following steps:

(i) univariate analysis aimed at explaining the frequency and distribution of sociodemographic characteristics of the respondents.

(ii) bivariate analysis conducted to examine simple associations between dependent and independent variables.

The data were entered into thematic matrices, through which the configuration of common patterns and differences were done.

\section{RESEARCH FINDINGS}

The Prevalence of HIV/AIDS in Yoruba Community: Delay in manifestation of AIDS long after the infection and the nature of the disease, which reflect the outbreak of many opportunistic infections account for inaccurate reporting of the cases of HIV/AIDS in the Yoruba community. In addition to those factors is the non-disclosing attitude of People Living with HIV/AIDS. The above factors are major hindrances to accurate statistical projection of HIV/AIDS prevalence in the community. Those factors also make the Yoruba people to contest the present official prevalent rates. Many of the people believe that 
cases of HIV/AIDS are not as rampant as it is claimed. A key-informant noted that the 'official prevalence rate is not true, because in this town I have not seen anybody infected with HIV/AIDS'.

Household structured interview was conducted in one local government selected from each of the research communities as the Enumeration Areas (EAs). In each of the EAs, two wards were selected where a total of 50 household heads were selected from each Local Government. A total of 200 household heads were randomly chosen from all the research communities. HIV/AIDS prevalence was measured. And there were only five households who reported cases of having one person each with identified symptoms of HIV/AIDS. Case study analyses of these people were conducted, and all of them were confirmed PLWAs. That implies that the prevalence rate is $2.5 \%$. This finding should be subjected to further interrogation; due to the hindering factors affecting the early manifestation of HIV/AIDS, as discussed above.

The official prevalence rates in Oyo and Osun States are very similar. Table 1 shows the prevalence rates of HIV/AIDS between 1980s and 2004.

Table 1: The official prevalence rates of HIV/AIDS in Osun and Oyo States between 1980 and 2004.

\begin{tabular}{lcc}
\hline Years & Osun State & Oyo State \\
\hline 1980 & $1.7 \%$ & $1.8 \%$ \\
1990 & $3.2 \%$ & $2.8 \%$ \\
2000 & $4.3 \%$ & $4.2 \%$ \\
2003 & $4.7 \%$ & $4.8 \%$ \\
2004 & $4.5 \%$ & $4.6 \%$ \\
\hline
\end{tabular}

Source: Oyo and Osun States' Ministries of Health, 2004.

Eighty-seven percent of the above cases were reported in urban communities with highest number of cases from Ibadan, Ilesa, Ogbomoso, Shaki, Ife, Osogbo, Ila-Orangun, Iwo, Ejigbo and Ede accordingly. Ibadan, Ilesa, Ogbomoso, Ile-Ife, Ila-Orangun and Ejigbo featured prominently in reported cases of HIV/AIDS between 1990 and 2003.

Only $13 \%$ of the reported cases are from rural communities. Rural communities are not accessible to HIV/AIDS test. Facilities for test are only found in urban hospitals. Thus, many cases are not reported from the rural areas. The implication of the above is that it is not valid to say that the prevalence of HIV/AIDS is urban-based.

There were more women infected with HIV/ AIDS than men. Seventy-three percent of the reported cases were women between 18 and 47 years old. Ninety percent of these cases were discovered during childbirth. Twelve percent of these cases were women outside matrimony. Out of this category, only ten percent were engaged in commercial sex work before the manifestation of the disease. It suggests that although commercial sex is susceptible to HIV/AIDS, being a risky sex, yet it is not the most vulnerable means to HIV/ AIDS prevalence in the Yoruba society. All the infected women have had sex either through premarital or/and marital sex. Eighty-eight percent of them claimed to have been faithful to their partners. But seventy-five percent of them further explained that their husbands were not residing with them, due to job placement to other communities; while the remaining eleven percent were widows and divorcees who remarried after loosing their initial husbands. This further confirms that the prevalence of HIV/AIDS in Yoruba community is due to male sexual permissiveness rather than female sexual permissiveness.

Women who engaged in risky sex do so unwillingly, but as a means of meeting their material needs. All the commercial sex workers confirm that they were not happy engaging in the act, but since the requirements of meeting their material needs push them into it, they then lack choice. There are some insignificant (3\%) numbers of women who engage in risky sex (extramarital) as a result of cultivated habit and not in need of any material gain. The benefit is psychological. This asserts the position that poverty draws women to risky sex, and not the desire of wanting to have children.

The prevalence of HIV/AIDS is thereby influenced by urbanization, women poverty and the breakdown of socio-cultural norms associated with sexuality in the Yoruba society.

Strategies against the Prevalence of HIVI AIDS: Influenced by the three global perspectives of HIV/AIDS, strategies against the prevalence of HIV/AIDS in the Yoruba society are:

(i) $\mathrm{ABC}$ Programme,

(ii) Use of Anti-Retroviral Drug,

(iii) Health education focusing on sexual and reproductive health rights,

(iv) Peer relation among PLWAs, and

(v) Advocacy.

ABC programme implies Abstinence from Sex, Be a faithful partner, and use of Condom. Advocacy against HIV/AIDS centers on this programme. The Yoruba people often detest the entire programme. 
The people believe that sexuality is a natural right, and that is a sign of well being. According to a key informant: "eniti kobobinrin sun kowaye re". Implying that he who does not engage in sex is not living well. Impotent persons are not regarded as living beings, because they can not procreate, and thus they are sick. Men and women who are weak sexually are also considered as being sick. That is why there exist a lot of herbs and concoctions to increase sex potency especially in men among the Yoruba people. Women also regard sex as one of the means to win men to fulfil women's wants and desires. Thus, based on the above, the people disregard the campaign on abstinence from sex. They believe that it is not practically easy to abstain from sex.

More women are faithful to their partners than men do; especially among the low literate population, and rural residents. It is a norm that women should be faithful to her husband. Thus ,Yoruba people are fond of saying that: "Oko mi ale elomiran". That is: 'My husband is mine, but he is a concubine to other women'.

Poverty and habits sometimes draw some women to be unfaithful to their partners, thus be involved in risky sex. In the case of adolescent pre-marital relationship, most adolescents keep multiple partners. For girls it is a practice, which enhances their chances of getting material things from boys, while for boys, it is a practice, which enhances their sociability and desires to have varieties of sexual taste and experience. These attitudes work to negate the use of condom. The use of condom is regarded as depriving men the utility of sex, thus many men feel reluctant to use condom. In the view of a key-informant (a woman) 'sexing with condom is like writing with covered pen'. Another key informant (man) noted that "sometimes one forgets to insert condom, especially when one is heightened with the desire to have sex'. All the PLWAs interacted with confessed their reluctance to use condom before infection. Also negating the use of condom is the perception that condom is costly, and that the promotion of condom has economic motives; else, there are many efficient local contraceptives which are not promoted.

Of recent, there is an introduction of AntiRetroviral drugs in the studied communities. Poor accessibility and its cost hamper the drug. Some PLWAs that were placed on the drug complained of its irregularity and cost burden. They are also not found in the rural areas at all.
Health education on HIV/AIDS only focuses on reproductive and sexual health. The idea centres on women empowerment to be able to defend their sex rights. Health education in this perspective is targeted to the vulnerable groups such as women and adolescents. This further explains the policy implication of the sex theory associated with cultural explanatory thesis of HIV/ AIDS. Health education on HIV/AIDS prevalence aims at discouraging risky sex, which is assumed to be emanating from women sex permissiveness.

Other strategies include peer-relation among the PLWAs, and Advocacy organized by NonGovernmental organizations. In the rural areas all these strategies are not popular, thus they failed to stem the prevalence of HIV/AIDS in the studied area. Seventy-two percent of the PLWAs interviewed maintained that it was after they had been infected when they learnt of those strategies.

\section{DISCUSSION}

HIV/AIDS is seen as a global danger in terms of material and human losses. Since its inception from 1980s, several millions of lives have gone into mortality, while many other millions of people are down with morbidity related to HIV/AIDS. Like malaria, maternal complications, tuberculosis and many other diseases, HIV/AIDS has great impacts on human and material development across all cultures. The understanding of disease and health is relative, thus factors responsible for morbidity and mortality in a particular disease vary from one society to another. This idea explains why community-specific approach is most favoured when conceptual analysis on health and development is undertaken.

Undoubtedly, there exist different cultural specifics on HIV/AIDS in African cultures, and there seems no basis for over-generalization of the perception of HIV/AIDS. The World Health Organization in 2003 has recognised this fact when the Organisation started to encourage local measures to avert the prevalence of HIV/AIDS, and care and support system for PLWAs. The Organisation is equally admitting the need to explore the local care to HIV/AIDS (Bloom et al., 2004).

The three global perspectives discussed in this paper have their inherent flaws to have given them a general application on all culture. It is true that HIV/AIDS is a global problem, and has similar aetiology, but certainly the social and cultural patterns of its aetiology are not the same in all 
human culture. This is simply because; the social and cultural settings in human societies are different too. Cultural explanation theory, which attaches the prevalence of HIV/AIDS on sexual permissiveness in women, undermines the fact that sexuality is a universal culture, but it is not conducted in the same way. There are many societies like the Yoruba people, where sexuality is guided with social norms, and the belief system. Among the Yoruba, sexual permissiveness is more exhibited by men than women. Men have control of their sexual rights and that of their daughters and wives.

Society has imposed incest taboos to discourage sexuality with blood related women. This is to protect the women sexual freedom from being abused by men. Similarly, in Yoruba society, sex is not free. There are accorded gratuities given to women by men in sex negotiation. The gratuity can be in form of money or material needs. This implies that women are "induced" for sex, and men should compensate women for consent to have sex. The socialization pattern protects women sexuality. Beginning from childhood, girls are taught to protect their sexuality by training them to dress decently, to sit down properly to keep their sexual organs from public view; not to stay with boys too often, and not to discuss sexuality in public. All these tend to repress the natural sexual permissiveness in women among the Yoruba people.

Although the prevalence pattern of HIV/ AIDS skewed towards transmission through sex, but the available data failed to confirm that there are more commercial sex workers among the women PLWAs in the region. Sexual permissivity among women, if exist, does not exist as a norm in the Yoruba society, rather it exists as a consequence of gender inequality which deprives women of access to community resources such as education, income earning probabilities, jobs, and other social needs (Ajala, 2003). In addition, women sexual permissivity also arises due to urbanization process, which has caused neglect of the rural communities. Many infrastructural facilities meant to support better living are located in the cities. Most of these are regarded as institutions, which support women inequality, and provide opportunity for many women to negotiate for community resources with men through sexuality. Such institutions include higher institutions of learning, food joints, big open markets, and big motor-parks. All of these institutions act as breeding places for itinerant sexuality. Pre-marital and extra-marital sexuality become more predominant in cities than the rural communities. Thus, the practice is vulnerable to HIV/AIDS. This position is against the cultural explanation by Odebiyi and Vivekananda (1991) which associates pre-marital and extra-marital sexuality to polygamy and African value for children. Of course quite a few of those engaging in such risk sex do it when they need children. In Yoruba culture both pre-marital and extra-marital sex relations are ideally considered as abnormalities. Thus, nobody does it and proclaims doing it publicly.

Dependency theory can only explain the mobility pattern of HIV/AIDS in the Yoruba society. Infection of HIV/AIDS has to do with rational choice made by individuals infected in terms of social needs. The individual choice to engage in risky sex, has no direct link with migration from rural communities, rather it has to do with individual attitudes in terms of what individual wants on migration to cities. Individual has choice to behave normal or abnormal. Without having to do with background setting. While it may be true that dependency explains rural poverty as a condition caused by urban dependence on rural community, thus, such can only explain the mobility pattern of HIV/AIDS and not the prevalence of HIV/AIDS. In the study area there is a high prevalence of HIV/AIDS in the cities, But dependency theory can not explain why it is so rather individual action leads to such prevalence. Social structures are in the cities to attract people into risky sex, not as a result of rural-urban drift; but certainly as a result of social actions, and derivation from symbolic interaction.

Rational Choice Explanation (RCE) seems to explain in part why individuals in the Yoruba society engage in risky sex. Individuals consider cost and benefit of either to be involved in risky sex or not. Although RCE explains why individuals engage in risky sex, but certainly HIV/AIDS infection is not restricted to risky sex alone. There are cases of infection arising from unrisky sex. For instance, some respondents $(75 \%)$ reveal that they were not involved in multiple sex, neither were they involved in unfaithful sex, but they were infected through legal and formal marriage with an infected partners who themselves were unaware of the infections.

The above discussions suggest that the Yoruba people of Southwestern Nigeria have 
specific cultural perspectives of HIV/AIDS prevalence. The society does not admit women sexual permissiveness as a norm, and in fact when it happens it is an aberration. It is even the males who are more sexually permissive than women, yet there are more cases of HIV/AIDS prevalence in women than men. There are more explanations to this rather than limiting the scope on women sexual permissiveness. The first explanation is that more women report to hospitals more than men do (Vulberruge, 1985). This is due to maternal health problems which women face. And HIV/AIDS infection is detected mostly in the hospitals usually when women go for maternal health problems. The second explanation is that stigmatization and rejection has more impact on men than women. Many men are infected, but refused to disclose because they do not want to loose their status.

As mentioned above, the implication of the three global perspectives on the prevalence of HIV/AIDS in the Yoruba culture can be summed in the following terms. (i) There is much emphasis on risky sex as being the cause of HIV/AIDS. (ii) In the society, women are more considered susceptible to HIV/AIDS, thus men see women as dangerous. (iii) Only cities are considered endemic to the prevalence of HIV/AIDS in Yoruba society. In addition, interventions against the prevalence of HIV/AIDS in the society focus discouraging risky sex; thus emphasis is on abstinence from sex, be a faithful partner, and the use of condom.

Due to the conceptual undermining of specific local perspectives of HIV/AIDS, the disease has further drawn the entire globe into more dependency on the international system. Developing countries depend on developed ones, and the rural communities depend on the cities to proffer solutions to the problems of HIV/AIDS. In the Yoruba community most of the actions against the disease have not optimally worked. It is difficult to abstain from sex, and equally difficult to determine and locate a faithful partner. Even faithful partners have also been affected. The use of condom is costly and the local alternatives are not encouraged. Thus, the HIV/ AIDS profiles continue to rise.

The present epoch of HIV/AIDS development is discovery/ intervention. Part of the discovery is that there is no generalized global perception of HIV/AIDS; rather attention should be directed to community-specific explanation. This approach takes into consideration, the specific knowledge, attitude, beliefs, and practices related to aetiology and etymology of HIV/AIDS in every society. From this perspective positive actions that will check the spread, provide better care and support system; and probably cure in HIV/AIDS will emanate.

HIV/AIDS is indeed a global problem, which has continued as a serious threat and tragedy in human society for over three decades. Its nature of spread has global perspective, yet there exists certain cultural specifics about the disease in all human culture. Therefore if a positive intervention is desirable, there is need to de-emphasize focus on global perspective of the disease. In the Yoruba society, the use of global perspectives to deal with the prevalence of HIV/AIDS has not positively worked.

\section{REFERENCES}

Ajala, A.S. 2003. "Cultural Context of HIV/AIDS in Yoruba Community: Implications for Care and Support for PLWAs." Unpublished Paper Presented at the International Conference on Better Care and Support for PLWA, in Dakar, Senegal, December 2003.

Ajala, A.S. 2004. "The Political-Economy of HIV/AIDS in Yoruba Society: Implications for Prevention, Care and Support in HIV/AIDS". Conference Proceedings, National Conference on HIV/AIDS, Abuja. May 2004.

Bloom, D.E., D.Canning and D.T. Jamison. 2004. "Health, Wealth and Welfare." Finance and Development, 2: 23-32.

Bloom, D.E., D.Canning and J. Sevilla. 2004b. "The Effect of Health on Economic Growth: A Production Function Approach." World Development 32: 1-13.

Cadwell, J.P., P. Cadwell and P. Quiggins. 1989. "The Social Context of AIDS in Sub-Saharan Africa." Population and Development Review, 15(1): 185-235.

FMOH. 2002. National Survey on HIV/AIDS in Nigeria. Abuja: Federal Ministry of Health.

Hunt, C. 1989. "Migrant Labour and Sexually Transmitted Disease" AIDS in Africa." Journal of Health and Social Behaviour, 6(2): 53-70.

NPC. 2001. Provisional Figures for National Population, Nigeria. Abuja: NPC.

Odebiyi, I.A. and F. Vivekananda. 1991. "AIDS in Third World Countries: Africa What are the Alternatives." Scandinavian Journal of Development Alternatives, $\mathrm{X}(1$ and 2): 91-99.

Osotimehin, B.2003. "1000 Nigerians Die Daily of HIV/ AIDS." The Punch, November $28^{\text {th }} 2003$.

Phillipson, T. and R.Posner (1995): "On the Microeconomics of AIDS in Africa." Population and Development Review, 21(4): 835-848.

Schoeff, B. 1992. "Women at Risk: Case Studies from Zaire", pp. 124-145, in G. Herdt and S. Lindenbaum (eds.) Social Analysis, Theory and Method. New York: Sage Beverly Hills (1992)

Verbrulggee, L.M.1985. "Gender and Health: An Update on Hypotheses and Evidence." Journal of Health and Social Behaviours, 26: 156-182 (1985). 